\title{
PARADIGMAS Y FUNDAMENTOS DE LA EVALUACIÓN EN EDUCACIÓN FÍSICA: RETROSPECTIVA Y PROSPECTIVA
}

\section{Roberto López Estévez}

1 Maestro de Educación Física. C.E.I.P. Leopoldo Alas, Madrid.

\section{lopez_roberto2004@yahoo.es}

\section{RESUMEN:}

El concepto de evaluación ha ido fluctuando en función del contexto social, histórico, político y educativo en el que se ha desarrollado.

En esta línea, hemos centrado nuestra atención en los modelos y paradigmas predominantes en las fuentes documentales consultadas, en relación a la evaluación; de esta manera, distinguiremos entre una vertiente técnica o tradicional y una vertiente práctica o alternativa, donde mostraremos como la Educación Física posee una idiosincrasia muy particular en función de una u otra.

Dentro de este marco, se exponen los fundamentos que sientan las bases de cada uno de los paradigmas de la evaluación en Educación Física desde un enfoque teórico, pedagógico y técnico.

PALABRAS CLAVE: Evaluación, Educación Física, Paradigmas, Fundamentos. 


\section{1.- CONCEPTO DE EVALUACIÓN}

El mundo heleno de la Edad Antigua es el precursor de utilizar la evaluación en el terreno educativo; así, filósofos como Sócrates utilizaban cuestionarios para evaluar a su alumnado. Algo parecido también ocurrió en la cultura romana.

En esta línea histórica, Forrest (1990) cita la remota práctica china en el siglo II a.C. con el objetivo de seleccionar funcionarios evitando las influencias de los grupos de presión de la burocracia como el origen de las prácticas selectivas de evaluación oral (citado por Gimeno y Pérez, 2008). También se hace eco de la exposición o defensa oral del alumnado ante sus profesores en las universidades de la Edad Media como práctica de evaluación académica (Beltrán y Rodríguez, 1994, citados por Hernández y Velázquez, 2004).

Por su parte, Blázquez (2003) hace referencia a la noción de evaluación; desde esta perspectiva elemental, Barlow (1992, citado por Blázquez y Sebastiani, 1998) atribuye la etimología de la palabra evaluación a los elementos latinos, originados en el Renacimiento, "ex"(fuera de), y "valoris" (valor).

En torno a este concepto, existe una cierta confusión y falta de consenso en relación al significado de la evaluación; López (2006) tilda a este hecho como "desconcierto terminológico" (p.36), debido a su carácter polisémico e ideológicopolítico.

La evaluación, por tanto, aparece en escena como una cuestión controvertida e integrada dentro del sistema educativo; estos planteamientos, también podemos apreciarlos dentro de nuestra área de conocimiento: la Educación Física. Existe un gran número de autores y autoras que corroboran estas ideas en relación a nuestra materia educativa (Fernández-Balboa, 2005; Hernández y Velázquez, 2004; López, 2000, 2006; López, Monjas y Pérez, 2003).

Nos encontramos, sin duda, ante un fenómeno que va a incidir de manera decisiva en el desarrollo del proceso educativo, condicionando a todos aquellos elementos que lo vertebran (objetivos, contenidos, competencias básicas, metodología, recursos,...). Es tal, la relación entre la evaluación y el proceso de enseñanza-aprendizaje, que se desencadena una especie de "encrucijada didáctica" (López, 2000, p.17), generando cierta correlación entre la evaluación y la práctica educativa: "dime como evalúas y te diré como enseñas" (López, 2000, ibíd.).

A lo largo del tiempo, son múltiples los autores que se han aventurado a dotar de significado a la evaluación; de esta manera, podemos citar los siguientes ejemplos: 


\begin{tabular}{|c|c|}
\hline AUTOR/A & DEFINICION \\
\hline Stufflebeam, D. (1971) & $\begin{array}{l}\text { "El proceso de delinear, obtener y suministrar } \\
\text { información válida para la toma de decisiones." } \\
\text { (citado por Chadwick y Rivera, 1991, p. 37) }\end{array}$ \\
\hline Nevo (1983) & $\begin{array}{l}\text { "Actividad que comporta, de manera inherente, el } \\
\text { hecho de emitir un juicio, independientemente del } \\
\text { objeto evaluado y de los criterios utilizados." (citado } \\
\text { por Blázquez y Sebastiani, 1998, p. 252) }\end{array}$ \\
\hline Rosales, C. (1984, p.11) & $\begin{array}{l}\text { "Reflexión crítica sobre todos y cada uno de los } \\
\text { componentes del sistema instructivo a fin de } \\
\text { determinar cuáles han sido, están siendo o serán los } \\
\text { resultados del mismo." }\end{array}$ \\
\hline $\begin{array}{l}\text { Chadwick, C. y Rivera, N. } \\
\text { (1991, p.36) }\end{array}$ & $\begin{array}{l}\text { "Análisis, consciente o inconsciente, de algo, para } \\
\text { emitir una opinión acerca de ese algo." }\end{array}$ \\
\hline González, M. A. (1995, p.11) & $\begin{array}{l}\text { "La interpretación mediante pruebas, medidas y } \\
\text { criterios, de los resultados alcanzados por alumnos, } \\
\text { profesor y proceso de enseñanza-aprendizaje en la } \\
\text { ejecución pormenorizada de la programación." }\end{array}$ \\
\hline $\begin{array}{l}\text { Diccionario de la Real } \\
\text { Academia Española de la } \\
\text { Lengua }\left(22^{\mathrm{a}} \text { ed.) }\right.\end{array}$ & "Acción y efecto de evaluar. Examen escolar." \\
\hline Sanmartí, N. (2007, p. 135) & $\begin{array}{l}\text { "Proceso de recogida y análisis de información } \\
\text { destinado a describir la realidad, emitir juicios de valor } \\
\text { y facilitar la toma de decisiones." }\end{array}$ \\
\hline Gimeno, J. (2008, p. 338) & $\begin{array}{l}\text { "Cualquier proceso por medio del que alguna o varias } \\
\text { características de un alumno, de un grupo de } \\
\text { estudiantes, de un ambiente educativo, de objetivos } \\
\text { educativos, materiales, profesores, programas, etc., } \\
\text { reciben la atención del que evalúa, se analizan y se } \\
\text { valoran sus características y condiciones en función } \\
\text { de unos criterios o puntos de referencia para emitir un } \\
\text { juicio que se relevante para la educación." }\end{array}$ \\
\hline Carbonell, J. L. (2012) & $\begin{array}{l}\text { "Proceso permanente de reflexión crítica sobre los } \\
\text { componentes y las interacciones en todo proceso } \\
\text { educativo, con el fin de descubrir los aciertos y } \\
\text { errores de dicho proceso, en la búsqueda de } \\
\text { soluciones más adecuadas para conseguir los } \\
\text { objetivos y fines que no hemos propuesto." (Paredes } \\
\text { y Herrán, 2012, p. } 375 \text { ) }\end{array}$ \\
\hline
\end{tabular}

Como se puede observar, son definiciones muy dispares en su contenido y que distan mucho en el tiempo unas de otras, por lo que podemos intuir que su significado va a estar condicionado en función del contexto histórico y socioeducativo del momento, y del paradigma en el que se integre. 
Desde mi punto de vista, y en base a las concepciones formuladas recientemente, entiendo que la evaluación debe responder a un proceso de enseñanza-aprendizaje, dentro de un contexto socioeducativo concreto, donde la información vertida debe ser analizada y valorada, con el fin de adoptar una serie de decisiones que nos permitan conseguir los fines propuestos.

Por tanto, para una mejor comprensión de las concepciones y aportaciones de los autores acerca de la evaluación, procederemos a delimitar dos posiciones antagónicas claramente diferenciadas; por un lado, la evaluación tradicional y por el otro, la evaluación alternativa:

\begin{tabular}{|c|c|c|}
\hline & $\begin{array}{l}\text { EVALUACIÓN } \\
\text { TRADICIONAL }\end{array}$ & $\begin{array}{l}\text { EVALUACIÓN } \\
\text { ALTERNATIVA }\end{array}$ \\
\hline Autores & $\begin{array}{l}\text { Tyler (1950), Stufflebeam } \\
\text { (1971), Lafourcade (1973), } \\
\text { Philips (1974), Torres } \\
\text { Santomé (1991), González } \\
\text { Halcones (1995), Sánchez } \\
\text { Bañuelos (1996). }\end{array}$ & $\begin{array}{l}\text { Gimeno (1981), Rosales } \\
\text { (1984), d ANÁLISIS prra } \\
\text { (1993), d y } \\
\text { Hernández y Velázquez } \\
\text { (2004), López Pastor } \\
\text { (2006). }\end{array}$ \\
\hline $\begin{array}{l}\text { Conceptos asociados a } \\
\text { la evaluación }\end{array}$ & $\begin{array}{l}\text { Sumativa, externa, vertical, } \\
\text { puntual, terminal, } \\
\text { heteroevaluación, } \\
\text { individual, postactiva,.. }\end{array}$ & $\begin{array}{l}\text { Formativa, } \\
\text { horizontal, } \\
\text { procesual, } \\
\text { dinámica, } \\
\text { comparticipada, }\end{array}$ \\
\hline Instrumentos & $\begin{array}{l}\text { Examen tradicional, } \\
\text { pruebas objetivas }\end{array}$ & $\begin{array}{l}\text { Pruebas de ensayo, de } \\
\text { elaboración y aplicación }\end{array}$ \\
\hline Profesorado & $\begin{array}{l}\text { Experto en conocimientos } \\
\text { académicos, } \\
\text { administrativo, ejercicio } \\
\text { técnico. }\end{array}$ & $\begin{array}{l}\text { Investigador, desarrollo } \\
\text { profesional, ejercicio ético, } \\
\text { implicación, compromiso. }\end{array}$ \\
\hline Alumnado & Pasivo & Participación activa \\
\hline Conocimiento & Aislado por disciplina & Interdisciplinar \\
\hline Enseñanza & Dirigida al examen & Dirigida a la comprensión \\
\hline Aprendizaje & $\begin{array}{l}\text { Centra su atención en } \\
\text { unidades discretas }\end{array}$ & $\begin{array}{l}\text { Atiende a la globalización } \\
\text { de los contenidos }\end{array}$ \\
\hline Otras características & $\begin{array}{l}\text { Eficacia, eficiencia, } \\
\text { rentabilidad, neutralidad, } \\
\text { conocimiento, aprendizaje, } \\
\text { ecuanimidad, objetividad. }\end{array}$ & $\begin{array}{l}\text { Calidad, equidad, } \\
\text { corresponsabilidad, } \\
\text { control, sanción, } \\
\text { imparcialidad, subjetividad. }\end{array}$ \\
\hline
\end{tabular}

En este sentido, la evaluación tradicional ha recaído sobre el alumnado, donde existían unos criterios precisos que daban lugar a emitir un juicio de valor y como consecuencia, valorar con una nota o calificación. El sistema educativo, está claro que no está compuesto sólo por el alumnado, por lo que el protagonismo también debería recaer en el profesorado y en el proceso. 
En su vertiente alternativa, la evaluación pretende en todos los casos y siempre, enriquecer, y si es preciso, mejorar las actuaciones futuras del alumnado y el profesorado, y por ende, del proceso educativo. La evaluación alternativa sugiere una serie de acciones, porque al ponerla en marcha, se consigue formar, explicar, educar, estimular, fortalecer, capacitar, comprender y reflexionar.

La evaluación intenta situarse en la cumbre del proceso educativo, ocupando un lugar estratégico y clave; en base a ello, Perrenoud (1990) afirma que:

La evaluación es un tema constante de preocupación de los docentes, de las autoridades escolares y de los investigadores de la Educación. Esto indica de por sí que se trata de un punto sensible, muy cargado afectiva e ideológicamente [...] Pero también muy rico en informaciones indirectas sobre las prácticas. (citado por López, 2006, p. 25)

Es loable que exista una tentativa al respecto como apunta Perrenoud, pero la realidad que se vivencia hoy en día en los centros docentes dista mucho de ubicar a la evaluación en un lugar privilegiado. Existe un profundo desconocimiento legal acerca de la evaluación, confundiendo los diferentes documentos en los que se encuentra inmersa (leyes, reales decretos, decretos, órdenes ministeriales, circulares, ...).

Con frecuencia, estos documentos de evaluación al igual que el currículo es visto por los profesores como un documento inútil, provocador de quebraderos de cabeza, o simplemente un mero trámite burocrático, sin ninguna utilidad real para el profesorado (Gómez et al. 2008)

Además existe una evidente desorientación de las prácticas evaluadoras por parte de los docentes, en muchas ocasiones, reproduciéndolas en el alumnado del mismo modo que les fueron aplicados a ellos cuando fueron estudiantes.

Entramos por lo tanto en un peligroso sin fin de despropósitos educativos, donde los errores y las consecuencias de los mismos se suceden sistemáticamente. En relación con esta nociva dinámica, dentro del área de Educación Física, Crum (1993) establece el "círculo vicioso autorreproductor de la E.F." (citado por Vizuete, 2002, p. 144), donde las ideologías convencionales monopolizan, en gran parte, la evaluación tradicional en los centros escolares.

Todas estas maneras de pensar, desarrollar y concretar la evaluación se cimentan sobre unos modelos y estructuras de conocimiento y pensamiento a través de los cuales la realidad cobra una determinada forma; estas percepciones e interpretaciones sobre el entorno se esconden bajo el paraguas de la racionalidad (López, Monjas y Pérez, 2003).

Estos enfoques pertenecen a un sistema complejo de conocimientos y creencias que se identifican con un determinado cuerpo doctrinal que se desenvuelve en marco concreto de referencia o paradigma. Este término, fue acuñado por Thomas Khun (1962) a través de su publicación The estructure of Scientific Revolutions y lo define como una completa constelación de creencias, valores y técnicas, compartidas por los miembros de una determinada comunidad (Fernández-Balboa, 2003). 

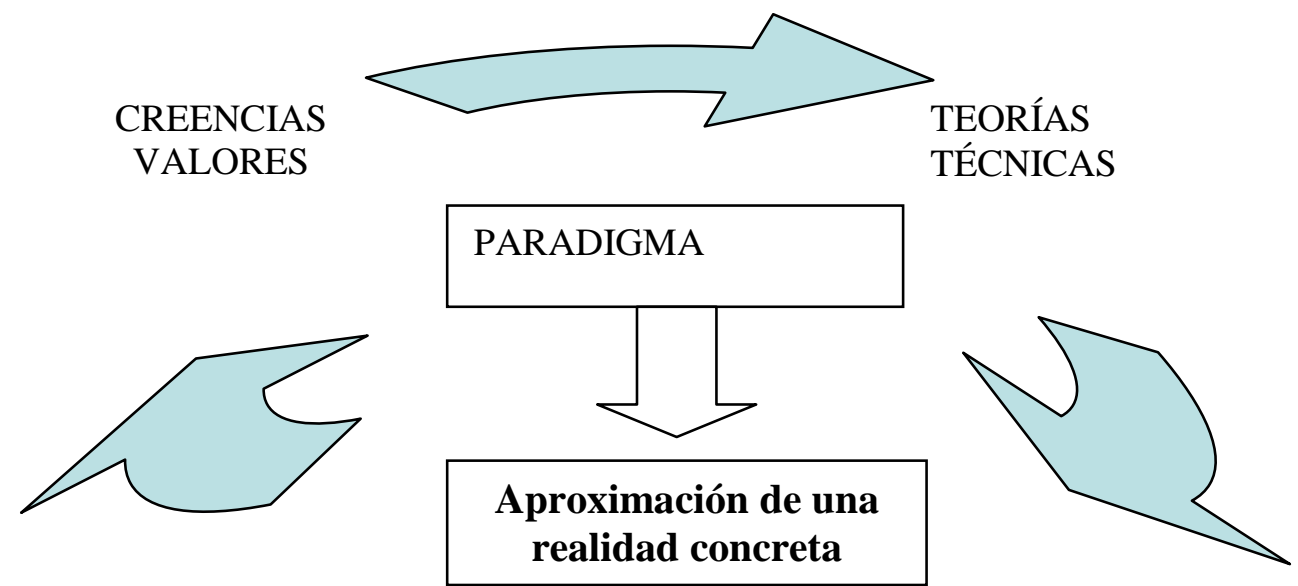

En base a esta cuestión, López (2006) sostiene que "la evaluación está estrechamente relacionada con las concepciones que se ponen en juego y con las teorías científicas y pedagógicas en que éstas se apoyan." (p.82); de acuerdo con este autor, podemos vislumbrar como dichas concepciones de la evaluación han ido evolucionando desde marcados procesos acentuados sólo en el aprendizaje y sus resultados, hacia la incorporación de la evaluación de los procesos de enseñanzaaprendizaje donde existe una interacción educativa (alumnado-contexto-profesorado), hasta la propia evaluación del proceso de enseñanza (planificación, programación,..).

De esta manera, todos los movimientos a favor de la evaluación que son sinónimo de cuantificación y medición con el objetivo de calificar o sancionar para obtener un determinado producto, se relacionan con una racionalidad técnica o instrumental (López, 2006), y englobados en un modelo de evaluación tradicional (Díaz, 2005) y un paradigma técnico, positivista o racional-tecnológico (López, Monjas y Pérez, 2003).

En el polo opuesto, las aportaciones de la evaluación que giran en torno a una valoración para la mejora de todos los elementos que intervienen en el desarrollo del proceso de enseñanza y aprendizaje, con el objetivo de retroalimentarse y optimizar el proceso, se relacionan con la racionalidad práctica o ética (López, 2006), dentro de un modelo de evaluación alternativa (Díaz, 2005) y amparado por un paradigma práctico, naturalista, interpretativo, crítico o sociocrítico (López, Monjas y Pérez, 2003).

\begin{tabular}{|l|l|l|}
\hline & \multicolumn{1}{|c|}{ TÉCNICO } & \multicolumn{1}{c|}{ PRÁCTICO } \\
\hline Teoría & $\begin{array}{l}\text { Universal, explicativa y } \\
\text { predictiva }\end{array}$ & Local, situacional y contemplativa \\
\hline Objetivo & Analítico, Empírico & Descriptivo y comprensivo \\
\hline Conocimiento & $\begin{array}{l}\text { Resultado de un proceso de } \\
\text { indagación y ausente de valor }\end{array}$ & $\begin{array}{l}\text { Construcción humana cargada de } \\
\text { valor, cambiante y contextualizada }\end{array}$ \\
\hline Realidad & $\begin{array}{l}\text { Sistema de variables } \\
\text { independientes }\end{array}$ & $\begin{array}{l}\text { Creada y mantenido por } \\
\text { interacciones simbólicas y pautas de } \\
\text { comportamientos }\end{array}$ \\
\hline
\end{tabular}

Una vez aclarado y precisado el conglomerado terminológico, pasaremos a dar respuesta a una serie de interrogantes que nos van a permitir desarrollar los diferentes paradigmas de la evaluación. En cada uno de los posicionamientos, partiremos desde 
el contexto socio-histórico en el que se enmarca y cómo ha influido cada uno de los modelos en el área de Educación Física, para posteriormente poder responder a las carácter más pedagógico ¿para qué, por qué y para quién evaluar? y otras cuestiones más técnicas que establece la evaluación ¿Qué, cómo, cuándo, quien y a quién evaluar?.

\section{PARADIGMA TÉCNICO}

\subsection{Evolución del paradigma técnico}

Este paradigma aparece descrito en la literatura bajo diferentes denominaciones; de esta manera, también es conocido con el nombre de paradigma positivista, tecnológico, normativo, cuantitativo, nomotético, proceso-producto (López, Monjas y Pérez, 2003, p. 51), racionalista, empírico-analítico, tradicional, (Soler y Vilanova, 2010, p. 27), es el que más tiempo se ha mantenido en el sistema educativo.

El paradigma positivista inicia su camino durante el s. XIX, en Estados Unidos, a través de la administración de evaluaciones por medio de test para analizar el rendimiento de las escuelas de Boston y el modelo educativo estudiantil (Morales, 2001).

Como podemos observar, estos procesos se basaban única y exclusivamente en los resultados, con el objeto de seleccionar en las escuelas a aquellos sujetos que fueran más idóneos para el sistema industrial. Este tipo de evaluación tecnicista tendrá sus repercusiones para afianzar el control, la planificación y la sistematización de las escuelas.

Durante la segunda mitad del s. XIX, comienza a florecer la filosofía del positivismo de Augusto Comte, con el objetivo de consolidar un único conocimiento válido que sirva como marco de referencia a través de la experiencia (Pérez, 2005). La influencia de Comte fue decisiva para el desmarque de métodos empíricos y estadísticos que van a cuantificar las capacidades del alumnado en las escuelas; así, por ejemplo, podemos citar el test de cociente intelectual de Stern (1912).

Con este tipo de pruebas, comienza una espiral de medición por parte de los psicólogos y de los docentes dentro del sistema educativo a través de baterías de test, lo cual acrecenta el interés por obtener resultados para ser contrastados y efectuar comparativas, sin tener ni las más mínima importancia el programa, la metodología, o mucho menos la labor del docente.

Hernández y Velázquez (2004), por su parte, apuntan al origen del paradigma técnico, a principios del siglo XX, dentro del terreno educativo como motor para satisfacer las necesidades de una sociedad industrializada, con el objetivo de racionalizar y tecnificar el trabajo.

En este momento, tras la I Guerra Mundial, la industria se erige como modelo dominante y organizativo de la producción y se pretender tomar al proletario como patrón para sistematizar el currículo escolar.

Este modelo de organización en la educación es defendido por Bobbit (1918) y se encuadra dentro de lo que Schiro (1978) llama la Ideología de la Eficiencia Social. Se pretende un profunda reforma en la escuela tradicional mediante un perspectiva utilitarista del currículo, donde el alumnado debe someterse a un entrenamiento para 
adquirir hábitos eficientes que se dirijan a la consecución de objetivos relacionados con actividades propias de una sociedad industrial (Gimeno, 1988).

No obstante, el paradigma técnico tiene su auge a partir de los año 50, con Tyler (1949) a la cabeza y su obra Principios básicos del curriculum y la instrucción (Pérez, 2005, p. 39) en el campo de la educación. Perpetúa el modelo cuantitativo y tecnocrático con el objetivo de medir los aprendizajes del alumnado y saber el grado de alcance obtenido.

Con ello, se otorga una importante operatividad a la programación a través de los objetivos; en este sentido, la evaluación cobra un gran protagonismo y es entendida como "el proceso que permite determinar en qué grado han sido alcanzados los objetivos educativos propuestos." (Morales, 2001, p. 168).

Tyler es considerado el verdadero padre de la evaluación sistemática, cuyo objetivo primordial es la comparación de resultados en función de un objetivos prediseñados. Dicha fórmula se centraba, exclusivamente, en el educando y en su rendimiento académico. El profesorado aparece en todo momento con una perspectiva mecanicista, pero con diferentes papeles: por un lado como un capataz en la función de control y vigilancia del alumnado, y por otro, como un experto que ofrece una serie de técnicas para hacer un determinado trabajo.

En nuestro país, se producirá una extensión del paradigma racional-tecnológico en la Ley 14/1970, de 4 de agosto, General de Educación y Financiación de la Reforma Educativa (Lorenzo, 1994, citado por Pérez, 2005) y continúa en los Programas Renovados de 1982 y en los Anteproyectos de 1985. Posteriormente, según el Ministerio de Educación (1989) habla de "evaluación puntual e integradora, uniforme o pluriforme, cualitativa, cuantitativa, interna, externa" (Álvarez, 2003, p.25).

A efectos de estas reformas y de acuerdo con Tiana (1990) la Ley Orgánica 1/1990, de 3 de octubre, de Ordenación General del Sistema Educativo (LOGSE en adelante), supone incorporar un significado más amplio al término de evaluación; desde este momento hasta nuestros días se hablará de evaluación de programas, de centros, de alumnado, de profesorado y del sistema educativo en su conjunto (citada en Hernández y Velázquez, 2004).

El discurso ofrecido durante el desarrollo de la evolución del paradigma racional-tecnológico, nos hace sentar las bases de este paradigma en "las Ciencias y la Tecnología" (Gimeno, 1998, p. 10) y apuntar como objetivo fundamental su idea de instrumentalizar y operativizar la educación para mantener y producir un sustrato social gobernado y regido por un modelo industrializado.

Podemos observar cómo se produce una despersonalización del alumnado y una alienación de la cultura educativa, en general, y del currículo en particular. Es un sistema que no responde a los problemas que presenta la educación o la sociedad, sino que tiene un beneficio puramente político-económico-empresarial. Gimeno (1988), muy acertadamente, acuña el término de "empresa escolar" (p. 16), comparando al escolar con el laboral, donde la máxima preocupación de este paradigma se reduce a una única frase, que se asemeja en la estructura al soliloquio de Hamlet: Ser o no ser eficaz.

\subsection{Enfoque curricular del paradigma técnico}

En base a los argumentos ofrecidos en el apartado anterior, se instaura un sistema educativo orientado a la producción y a la consecución de objetivos de una 
forma eficaz, donde el currículo va a poseer un carácter cerrado y eminentemente técnico.

Las teorías conductistas hunden sus raíces en este paradigma y van a tener una incidencia muy significativa en el currículo. En el terreno educativo va a ser Skinner, el padre del condicionamiento operante, el que adquiera una mayor relevancia.

El proceso de aprendizaje va a estar mediatizado por la asociación de estímulos, respuestas y recompensas (positivas y negativas), con el objetivo de alcanzar una eficaz conducta en la consecución de objetivos a corto, a medio y a largo plazo.

Las directrices a las que Skinner pretende someter al alumnado se basan en "programas de refuerzo, la enseñanza programada, las máquinas de enseñar, los programas de economía de fichas en el aula, el análisis de tareas, los programas de modificación de conductas,..." (Gimeno y Pérez, 2008, p. 39)

Por tanto, surge la necesidad de crear un modelo curricular eficiente que dé respuesta a las necesidades de los sistemas de producción industrial.

Gimeno (1984) apunta hacia una unidireccionalidad del currículo, convirtiéndose en un objeto de gestión y perdiendo completamente su carácter histórico, social y cultural (citado por Pérez, 2005).

Tras estos prolegómenos, Inlow (1966, citado por Pérez, 2005) nos aporta una definición de currículo asociado a un proceso técnico como en el que nos encontramos; así, lo conceptualiza como un "esfuerzo conjunto y planificado de toda escuela, destinado a conducir el aprendizaje de los alumnos hacia resultados de aprendizaje preestablecidos." (p. 41)

Al respecto, Gimeno (1991) afirma: "Desde una perspectiva interna escolar, el currículum enfatizado es el seleccionado de hecho como contenido de los procedimientos de control" (citado por Bores y Escudero, 1997, p.154).

Dicha sistematización curricular responde al nombre de currículo por objetivos (Díaz, 2005) y es propio de un discurso de rendimiento dentro de una programación por objetivos (López, 2006) o una pedagogía por objetivos (Gimeno, 1988; Pérez, 2005).

Los elementos imperantes del currículo, sin lugar a dudas, son los objetivos; el poder que se le otorga a los objetivos para obtener resultados, hace que el resto de elementos curriculares queden relegados a un segundo plano. Al respecto, Díaz (2005) afirma que los objetivos deben formularse a través de comportamientos que sean susceptibles de observación y medición.

Nos encontramos, por tanto, como establece Kirk (1990) ante un "enfoque centrado en la conducta del alumno y claramente vinculado con una preocupación por rendir su rendimiento, el cual se considera como indicador de efectividad de la enseñanza y la calidad de un programa educativo." (p. 123)

Toda enseñanza o programa educativo tiene una serie de intenciones y finalidades que se han de plasmar a través de unos propósitos; éstos no son, ni más ni menos, que los objetivos que se pretenden alcanzar al finalizar un proceso de enseñanza-aprendizaje. 
Actualmente, los objetivos de carácter general se van diseccionando en otros más concretos (objetivos de área, objetivos de ciclo, objetivos de curso, objetivos didácticos,...); pero, las exigencias de cuantificación y control que impone el paradigma racional-tecnológico, provoca que los objetivos generales se traduzcan, según Ordoñez (1990) en "objetivos operativos o conductuales" (p. 92), que funcionan como una herramienta docente para medir y obtener resultados.

Estos objetivos, a su vez, están organizados y jerarquizados en términos de habilidades, destrezas y capacidades a través de las taxonomías; dentro de esta línea conductista, cabe citar las taxonomías de Bloom (1971) y Gagné (1979) con un corte muy psicológico y separándose de los términos de ejecución que proponían Popham (1970), Tyler (1973), etcétera. (Ordoñez, 1990).

Tanto Bloom como Gagné han sido un importante punto de referencia en los aprendizajes de la Educación Física; sus taxonomías, de corte "militar e industrial" (Gimeno, 1988, p. 40) han aparecido en numerosos manuales de Educación Física para el profesorado, en temarios de oposiciones a nuestra especialidad, en apuntes de formación del profesorado,...considerándose un elemento esencial para organizar la tarea educativa.

Por tanto es indiscutible, que la Educación Física se enfoca hacia el adiestramiento, la instrucción, el adoctrinamiento y la ejecución; a partir de Bloom, se comienza a hablar de los objetivos "cognoscitivos, afectivos y senso-motores" (Ordoñez, 1990, p. 99), pero realmente, sólo le dé da importancia a los objetivos cognoscitivos que son los que más influencia y tradición han tenido en el terreno académico.

La evaluación desde un curriculum técnico, cómo el que se acaba de describir, está sometida a un rigor burocrático en la medición de la adquisición de conocimientos para, valorar, sólo y exclusivamente, el producto final.

González (1995) explicaba que "evaluar implica ineludiblemente medir. Hemos de recurrir a la medición como elemento indispensable de la evaluación" (citado por López, 2006, p.44). En base a esta reflexión, habría que destacar que la evaluación educativa, en general, recurre a esta técnica para comprobar el rendimiento académico del alumnado y ponerlo en conocimiento de la Administración educativa.

Los propósitos fundamentales no se encaminan hacia la evaluación, sino hacia la calificación del alumnado en base a unos resultados mensurables.

El esquema estímulo-respuesta que propone el conductismo, enfoque principal en este paradigma, no es ni de lejos la solución a los problemas que aparecen dentro de un grupo-clase, por lo que no deja de ser un modelo básico que no tiene en cuenta las interacciones entre el grupo humano, el medio en el que se desenvuelven y las situaciones cambiantes que se suceden.

Este modelo por tanto se traduce y se reduce a un solo componente; es decir, no desarrolla otros potenciales y capacidades que son inherentes a la naturaleza del ser humano; más bien, es un modelo qué no se preocupa en absoluto, de aquello qué es capaz de aprender por el individuo.

\subsection{La evaluación en Educación Física dentro del paradigma técnico}

\section{- Fundamentación teórica}


Este modelo de evaluación ha sido predominante durante los últimos 40 años y el más extendido en el área de Educación Física. Responde a aspectos marcados de forma cuantitativa y conductista, buscando la medición y eficacia, sin darle mayor importancia al aspecto cognitivo, formativo, pedagógico o educativo. Imbernón (1992) resalta que la Educación Física también cumple otros objetivos educativos, fuera del plano motor.

Este planteamiento se contrapone a los principios pedagógicos que establecen las disposiciones legales en Educación, y suele generar un visión escolar relacionada única y exclusivamente con la educación corporal y motriz, sin abarcar los demás ámbitos de la personalidad (afectivo-emocional, social, psicológica,...)

Desde una perspectiva tradicional la evaluación de la Educación Física según Bloom (1970) ha estado sometido a un "currículum por objetivos" (López, 2006, p. 47). De dicho curriculum, puede verse reflejado como la Educación Física queda reducida a un área estrictamente instrumental, donde el cuerpo ha sido comparado a una máquina, donde se pone el énfasis en la comprensión puramente anatómica y fisiológica del cuerpo humano, en la medición de resultados y la preocupación por la mejora en la ejecución técnica y en la condición física.

Smith (1972) analizó está problemática, criticando que "no viene al caso plantear la Educación Física como algo opuesto, o que se añade, a la educación moral o intelectual. Solamente existe un tipo de educación, esto es, una educación integral de la persona" (citado por López, 2006, p. 48)

El modelo técnico se va a centrar por tanto en las capacidades motrices del individuo que persiguen un objetivo determinado y fácilmente medible; esto nos da pie, a destacar que sólo se desarrollan aquellas capacidades relacionadas con el ámbito físico-motor, dejando de lado el plano cognitivo y socioafectivo.

La Educación Física se configura para un alumnado con una buena condición física, sin tener en cuenta el nivel de partida ni las experiencias motrices anteriores. Por ello, no existe ningún tipo de atención a la diversidad, donde todo pasa por ensalzar y atender a aquellos alumnos que por motivos filogenéticos, antropométricos o patológicos poseen unas habilidades motrices más desarrolladas sobre aquellos que no tienen un adecuado bagaje motor.

López y Fontana (1998) nos exponen diferentes ejemplos prácticos en los que se justifica el incumplimiento de las medidas de atención a la diversidad y la desigualdad que generan aquellos condicionantes que no son modificables:

\begin{tabular}{|l|l|}
\hline PRUEBA & JUSTIFICACIÓN \\
\hline Lanzamiento de balón medicinal & $\begin{array}{l}\text { En función de la altura del alumnado, la } \\
\text { salida del balón provocará diferente } \\
\text { alcance con la aplicación de una misma } \\
\text { fuerza }\end{array}$ \\
\hline $\begin{array}{l}\text { Test de Wells (flexión de tronco } \\
\text { adelante desde sedestación) }\end{array}$ & $\begin{array}{l}\text { El resultado variará según los parámetros } \\
\text { antropométricos de los miembros } \\
\text { inferiores o superiores }\end{array}$ \\
\hline Test de Cooper (resistencia aeróbica) & La genética puede hacer que el resultado \\
\hline
\end{tabular}




\section{0 metros lisos (velocidad)}

esté muy determinado (por la mayor o menor proporción del tipo de fibras que influyen en el rendimiento de la prueba)

Esto acarrea consecuencias negativas en el alumnado menos habilidoso, pudiendo provocar una merma en la autoestima, una falta de progresión en sus capacidades y habilidades motrices, una desmotivación y animadversión hacia la asignatura o el profesorado....En este sentido nos encontramos ante una Educación Física enfocada hacia el rendimiento motor, sin hacer hincapié en la educación en valores, la salud, la expresión corporal, la coeducación, etc. y por supuesto, donde el alumno no percibe, ni piensa ni decide, sólo se limita a ejecutar. Se sobreentiende, por tanto, que el profesorado aparece como una figura que no estimula el desarrollo cognitivo-motriz a través de la reflexión y la comprensión.

De esta forma, la Educación Física subyace bajo la influencia del paradigma positivista y con un diseño curricular programado por objetivos orientados hacia el rendimiento y la eficacia (tiempo de actividad motriz, cantidad de feedback,...); Velázquez y Hernández (2005) señalan como este fenómeno ha ido deteriorando la vertiente socioeducativa y pedagógica de esta área curricular (citado por López et al., 2007, p. 63).

La ideología del rendimiento focaliza su atención sobre el deportista de élite y el alumnado más capacitado (Devís y Pérez-Samaniego, 2009). Nuestra intención no debe ser, ni mucho menos, formar a futuros campeones ni desarrollar programas deportivos orientados al entrenamiento y la competición, ya que este tipo de objetivos deben quedar desterrados fuera del terreno escolar.

\section{- Fundamentación pedagógica}

La cuestión principal a resolver es: ¿Para qué evalúa el modelo tradicional de la Educación Física?.

Sus principales finalidades son el rendimiento físico y la selección de talentos deportivos. Este modelo responde a funciones sancionadoras y de control, cuando no de selección, convirtiendo el proceso de evaluación en fuente de calificaciones, para conseguir unos resultados dentro de un determinado período temporal y en función de una serie de criterios de evaluación inflexibles y dirigidos a valorar una conducta medible y predeterminada.

Dichos propósitos ponen de manifiesto los argumentos de Fernández y Navarro (1989), quienes sostienen que: "La evaluación en Educación Física ha sido mal utilizada pedagógicamente, ya que han prevalecido una evaluación sumativa en base a la aptitud física." (citados por López, 2006, p.55)

Todo ello responde a un carácter fuera de la educación integral de la persona, y reduce el proceso de evaluación a simples esquemas de numeración y puntuación. De esta manera tan cuantificada, Fernández (2005) critica que: "cuando se reduce a las personas a números, se las despersonaliza y, una vez despersonalizadas, se las puede oprimir y eliminar con mayor facilidad" (p.132).

En este sentido, no se percibe el menor atisbo de la evaluación del proceso de interacción profesorado-alumnado y mucho menos la evaluación de la labor del docente. Se evalúa, por tanto, para determinar si el alumnado ha conseguido los 
objetivos predeterminados y marcados en el currículo; y posteriormente, se califica en función de la consecución o no de dichas conductas.

De esta manera, López (2006) alude a esta valoración como evaluación técnica y la relaciona con el área de Educación Física en los siguientes términos "medición, control, calificación y poder" (p. 84)

Paralelamente, responderemos al ¿por qué evaluar? conectando con las afirmaciones arrojadas en las finalidades de la evaluación en este modelo técnico.

En este sentido, la evaluación sigue cumpliendo funciones encaminadas a medir, controlar, calificar y de poder, generando un modelo, mecánico y reproductor, según Miller (1980) de "pedagogía venenosa" (citado por Fernández-Balboa, 2005, p.130).

Se perpetúa por tanto una práctica docente, que por un lado viene determinada por la ideología del rendimiento y la eficacia, pero también como señalan Devís y Pérez-Samaniego (2009) aparece una reacción contraria orientada al "recreacionismo" (p. 115) donde la actividad física sirve como descanso, recreo, tiempo libre,...

Este tipo de evaluación objetiva va in crescendo en las diferentes etapas educativas, lo que va complicando de manera significativa el avance hacia planteamientos funcionales y educativos, fomentando una falta de interés y compromiso profesional en la educación.

De esta manera, entonces, ¿para quién se evalúa?. La evaluación queda en manos de un sistema educativo político y burocrático, donde el saber es tratado como moneda de cambio, para lo cual necesita ser reducido a categorías fácilmente medibles (Álvarez, 2003).

Por tanto, quien se beneficia de esas categorías prefabricadas y estandarizadas es el profesorado, lo cual le aporta un confort didáctico y metodológico. Para ello las editoriales ofrecen numeroso material didáctico para el área de Educación Física, el cual no debe tomarse como una "biblia" para el profesorado, sino como una herramienta complementaria para desarrollar el proceso de enseñanzaaprendizaje.

\section{- Fundamentación técnica}

¿Qué evaluar?. Para ello, tomaremos como referencia a Chaparro y Pérez (2010) quienes centran el objeto de evaluación en las acciones demostradas por los alumnos; es decir, muestran una línea estrictamente procedimental en las cuestiones relativas al aprendizaje.

$\mathrm{Ni} \mathrm{el} \mathrm{profesorado} \mathrm{ni} \mathrm{los} \mathrm{demás} \mathrm{agentes} \mathrm{socioeducativos} \mathrm{aparecen} \mathrm{en} \mathrm{escena} \mathrm{a}$ la hora de ser realizada la evaluación. Todo el peso de la evaluación recae sobre el alumnado en función de unos criterios relacionados con unos objetivos conductuales que ponen el énfasis en la disciplina y en la ejecución de las tareas.

En cuanto a los contenidos a evaluar, fundamentalmente se focalizaba la atención en las capacidades físico-motrices (fuerza, velocidad, resistencia y flexibilidad) y en las habilidades y destrezas motrices orientadas a los deportes convencionales (fútbol, voleibol, atletismo,..) bajo una metodología directiva, analítica y repetitiva, propia de los modelos de entrenamiento deportivo. 
Respecto al ¿Cómo evaluar? la conceptualización de la evaluación tradicional en Educación Física, tiene su origen en la aplicación de las Ciencias Biológicas y en el rendimiento deportivo. Las pruebas y los test físico-motrices guardan una estrecha relación con las áreas biomédicas, como la fisiología, la anatomía y la biomecánica, principalmente (López, 2006).

Se han basado prioritariamente en la realización de test y pruebas asociadas al rendimiento motor y a la mejora de la técnica físico-deportiva, con la única pretensión de calificar al alumnado. Blázquez (1993) explica como la psicometría sirve de modelo para inspirar los criterios de evaluación en Educación Física (citado por López, 2006).

Entre los diferentes instrumentos de evaluación objetiva podemos encontrar escalas, registros, perfiles psicomotores, pruebas estandarizadas (p.e: batería Eurofit),...configurados en función de una escala de medida que corresponde con unas puntuaciones y unos resultados predeterminados.

No obstante, de acuerdo con Blazquez (1994) habría que clarificar que los datos obtenidos mediante esta serie de pruebas puede tener un sentido práctico y funcional, a través de una fase de diagnóstico y de valoración de las cualidades motrices con el fin de alcanzar estilos de vida saludables (citado por López y Fontana, 1998).

En este sentido, Díaz (2005) critica que:

La racionalidad técnica fundamentada en una perspectiva cuantitativa y en la búsqueda de la máxima objetividad ha hecho que algunos de estos instrumentos se conviertan en pruebas de laboratorio, las cuales no responden a la realidad educativa y escolar en los procesos de enseñanza aprendizaje. ( $p$. 142)

Esta dinámica viene siendo un continuo en la Educación Física actual en los diferentes centros docentes y de formación, donde las condiciones del entorno y del alumnado son pasadas por alto y se produce la aplicación de los instrumentos sin una progresión ni una significatividad en las tareas de evaluación y en el aprendizaje. Por ello, la percepción del alumnado sobre la calificación se relaciona con el resultado obtenido en la aplicación de los instrumentos de evaluación. Blázquez (1990), en este caso, habla de evaluación referida a la norma.

En base a estas afirmaciones, no podemos permanecer impasibles ante la posibilidad de que un proceso de aprendizaje se despersonalice por el mero empleo de un mecanismo calificador estandarizado que no responde ni tiene en cuenta a las características psicoevolutivas y ni el contexto en que se desenvuelve el alumnado. Todo se reduce a un aislamiento del discente y a someter a su aprendizaje en momentos concretos del período escolar, bajo el mando y el dominio imperante de las herramientas docentes para cuantificar, seleccionar y calificar.

En relación al ¿cuándo evaluar? desde un prisma técnico o tradicional de la evaluación en el de Educación Física, hemos de centrarnos en un momento crucial y concreto; dicho espacio de tiempo se convierte en un área de acción destinada a establecer resultados finales y calificarlos en base a unos estándares previamente establecidos.

De esta manera, podemos identificar claramente una evaluación sumativa o final (Chaparro y Pérez, 2010), donde al término de la prueba o de la una unidad de trabajo se ponen sobre la mesa los resultados obtenidos, sin atender al resto de 
momentos cumbres que forman parte de ese proceso de evaluación en la unidad didáctica.

Para abordar el siguiente interrogante ¿a quién evaluar? entendemos que el criterio dominante en este caso es la unidireccionalidad, ya que la evaluación se centra exclusivamente en el alumnado, desechando así el proceso de enseñanza. De esta manera, podemos hablar de una heteroevaluación, donde la figura del profesor de Educación Física representa un modelo hegemónico en la toma de decisiones en el proceso de evaluación.

La coevaluación se centra exclusivamente en el desarrollo de la condición física y se da sólo entre alumnos; aparece bajo un diseño muy cerrado por parte del profesorado, donde éste se limita a dar instrucciones al alumnado de quién, cómo y cuándo tienen que hacerlo, sin hacer hincapié en el qué, para qué y el por qué. Este tipo de coevaluación tiene un objetivo fundamental: recoger datos para luego calificar en base a unos baremos estandarizados.

Por tanto, el alumno pasa a ser un mero observador y registrador de informaciones, sin comprender la naturaleza de la tarea y del proceso.

\section{PARADIGMA PRÁCTICO}

\subsection{Evolución del paradigma práctico}

El paradigma práctico, también responde a otros nombres como cualitativo, fenomenológico, naturalista, humanista o etnográfico (Soler y Vilanova, 2010, p. 28), interpretativo, interpretativo-simbólico (Pérez, 2005, p. 87), hermenéutico (Gimeno y Pérez, 1985, p. 91), ecológico, ideográfico (López, Monjas y Pérez, 2003).

Para desarrollar el origen de este paradigma nos apoyaremos en Pérez (2005), quien sostiene en su obra que durante la década de los años 70, Schwab (1983) inicia un movimiento contrario al paradigma racional-tecnológico, con el fin de analizar, comprender y reflexionar sobre la práctica educativa con una intención clara de mejora en la pedagogía ejercida.

Este golpe de efecto, tiene su repercusión en Inglaterra a través de Elliot y Stenhouse, y también en nuestro país, con Gimeno Sacristán que presenta en su libro "La pedagogía por objetivos. Obsesión por la eficacia" en 1982, en el cual describe y critica los fundamentos del paradigma tradicional y aporta alternativas contrarias al modelo hegemónico.

Este movimiento de renovación pedagógica considera la realidad social y educativa, no como algo inmutable, sino como un conjunto de variables complejas y dinámicas, donde el protagonismo no debe limitarse a la enseñanza, sino también al aprendizaje y a los procesos de interacción que ocurren entre ellos.

El paradigma práctico, establece un profundo cambio en la concepción curricular, donde los objetivos no se refieren a comportamientos mensurables, sino que se centra en todos aquellos elementos que componen el proceso de enseñanza y aprendizaje, dándoles forma e integrándolos en la evaluación.

Por tanto, la evaluación se sustenta sobre un complejo multidimensional donde cada uno de los elementos que configuran el sistema educativo debe estar interrelacionados y al servicio del proceso de enseñanza y aprendizaje. 
En relación a esta conclusión, cobra especial relevancia la evaluación formativa, la cual debe entenderse, según Álvarez (2003) como "aquella evaluación puesta al servicio de quien aprende, aquella que ayuda a crecer y a desarrollarse intelectual, afectiva, moral y socialmente al sujeto" (p.116).

En su intención formativa, la evaluación pretende en todos los casos y siempre, enriquecer, y si es preciso, mejorar las actuaciones futuras del alumnado y el profesorado. La evaluación formativa sugiere una serie de acciones, porque al ponerla en marcha, se consigue formar, explicar, educar, estimular, fortalecer, capacitar y perfeccionar.

Santos (1993) expresa que para conseguir que una evaluación sea formativa se tiene que sustentar en un proceso de diálogo, comprensión y mejora (citado por Díaz, 2005).

El carácter formativo de la evaluación se utiliza para tratar de mejora el proceso de enseñanza y aprendizaje a partir del conocimiento que genera sobre la forma en que transcurre dicho proceso y sobre el grado de adecuación y calidad de las interacciones que se dan entre los diferentes elementos que intervienen en el mismo (Hernández y Velázquez, 2004).

Tras esta renovación pedagógica del sistema de evaluación en el terreno educativo, aparecen diferentes término asociados a este concepto de evaluación formativa; así encontramos la evaluación alternativa, la evaluación innovadora, la evaluación auténtica, la evaluación para el aprendizaje y orientada al aprendizaje, y la evaluación formadora (López, 2009)

Estas concepciones de la evaluación se encuentran orientadas a mejorar el aprendizaje del alumnado y capacitar al individuo hacia la autorregulación de su propio proceso de aprendizaje y que se produzca una transferencia positiva del mismo en su vida cotidiana.

Llegados a este punto, podemos observar como el sujeto que aprende pone en marcha una serie de mecanismos para desarrollar y regular todo ese período donde se vierten los diferentes aprendizajes, configurándose un proceso de autonomía personal.

En este momento, se alberga la idea de plantear una estrategia de evaluación dirigida a promover la autorreflexión y el control sobre el propio aprendizaje. En función de este planteamiento, Nunziati (1990) atiende a la evaluación formadora como un sistema pedagógico donde la autoevaluación es la piedra angular para construir un modelo de ciudadano autónomo, crítico, responsable y participativo de una sociedad democrática (citado por Hernández y Velázquez, 2004).

Díaz (2005) establece que la diferencia de la evaluación formadora frente a la evaluación formativa se desprende en el mayor nivel de autonomía que se le ofrece al alumnado a la hora de controlar y desarrollar su aprendizaje.

En este caso, para encontrar un marco legislativo coherente con un planteamiento práctico tenemos que trasladarnos a la última década del siglo XX con la implantación de la Ley Orgánica 1/1990, de 3 de octubre, de Ordenación General del Sistema Educativo (LOGSE); en este sentido, se hace alusión a la función pedagógica que supone el correcto funcionamiento de los procesos de enseñanzaaprendizaje; al respecto, Contreras (2009) coincide con "lo que supone evaluar la metodología, las estrategias, los objetivos propuestos y su grado de consecución, los 
contenidos, los materiales empleados y las actividades desarrolladas para adecuar la acción del profesorado" (p. 297).

El cambio más significativo y que más se ha analizado hasta el momento actual, son las competencias básicas propuestas por la Ley Orgánica 2/2006, de 3 de Mayo, de Educación (LOE); desde un punto de vista transversal, se pueden considerar como un eje común en las enseñanzas europeas, y al poseer un carácter abierto y flexible, favorece el desarrollo de las competencias.

Por tanto, al sumergirnos dentro de este paradigma deberemos tener presentes "los parámetros evocados en esa imagen a partir de un sujeto que piensa, siente, actúa y se relaciona en un contexto socio-cultural determinado." (Minkévich, 2009, p. 290); de acuerdo con este autor, nos encontramos a la evaluación en un escenario configurado por los diferentes agentes sociales y educativos (alumnado, profesorado, familia,..) dentro de un entorno fluctuante e interrelacionado donde surgen diferentes procesos de acción.

\subsection{Enfoque curricular del paradigma práctico}

El enfoque curricular del paradigma práctico está basado en la idea de proceso; éste trata de considerar la complejidad de los problemas y las teorías en el que se encuentra sustentado el sistema educativo (Pérez, 2005).

Desde el paradigma práctico aparecen nuevas definiciones acerca del currículo, entre las que cabe destacar la aportada por Stenhouse (1984) como elemento que "debe constituirse, dentro de la teoría práctica, como un proyecto que se verificará en un proceso práctico, real y concreto." (p. 29); más adelante, Grundy (1991) defiende que el currículo es una construcción sociocultural y no un mero concepto, y lo conceptualiza como "una forma de organizar un conjunto de prácticas educativas humanas." (p. 32)

El interés se centra en la calidad del proceso educativo entendiendo el currículo desde un enfoque de racionalidad práctica. Esta perspectiva curricular concibe el currículum como "un proyecto y un proceso" (López, Monjas y Pérez, 2003, p 54).

El curriculum desde una racionalidad práctica, entiende la evaluación como un proceso y un proyecto centrado en el diálogo, la comprensión y la mejora, para la construcción del conocimiento. Así, la evaluación cumple una función fundamentalmente epistemológica, además de educativa, que pone a quienes enseñan y a quienes aprenden en relación con el conocimiento (Álvarez, 2001).

Un curriculum abierto y flexible como el que define nuestro sistema educativo lleva inherente la necesidad de que alguien lo tenga que cerrar o concretar en función de diferentes factores. El profesor es la persona que tiene la llave para regular ese diseño curricular y adaptarlo a la realidad educativa vigente.

El docente aparece en escena como un investigador de la acción en el aula y analista de las respuestas del alumnado para reformular y readaptar el proceso a las necesidades de éstos (Pérez, 2005).

Estos procedimientos se fundamentan en unos pilares psicopedagógicos aportados en su momento por autores como Piaget, Inhelder, Bruner, Flavell, Ausubel,... son los representantes de la psicología cognitiva; donde los resultados obtenidos de esta corriente son fundamentales para entender el aprendizaje humano 
(Pérez y Giménez, 2008). También se construyen en procesos de intercambio. Por ello, se denomina a estas posiciones como "constructivismo genético" (p. 43).

En contraposición al conductismo, Piaget considera que el sujeto debe ser capaz de ofrecer una respuesta en función de las circunstancias en las que se encuentra organizado el entorno.

El modelo educativo que se desprende de este paradigma interpretativo es de corte constructivista, donde los objetivos fundamentales se orientan hacia el proceso de enseñanza y aprendizaje en el que interaccionan los diferentes agentes socioeducativos.

El concepto de educación en este modelo responde a una necesidad social, y no a una necesidad político-económica como en el paradigma técnico, ya que el individuo no se desarrolla sólo de forma personal, sino también de manera social (Hernández y Velázquez, 2004).

La educación, por tanto, es entendida como un proceso de desarrollo de capacidades a través de una serie de actividades en diferentes ámbitos de convivencia, y así queda reflejada en Pérez (1995) cuando afirma que:

La educación debe formar a las personas como miembros de una ciudadanía de forma activa y autónoma en la construcción y desarrollo de la sociedad democrática...mediante el ejercicio de la reflexión crítica y autocrítica y de la participación activa y cooperativa en el escenario social del aula y de la institución escolar. (citado por Velázquez, 2000, p. 483)

A través de este modelo, se quiere desarrollar un sistema de autogestión del alumnado, donde éste tendrá que tener una capacidad indagadora para obtener información; además, deberá ser crítico para analizar y discernir qué elementos son los que mejor se adecúan a su objetivo; todo ello le dará pie a tomar decisiones que desarrollarán su propio proceso de aprendizaje.

\subsection{La evaluación en Educación Física dentro del paradigma práctico}

\section{- Fundamentación teórica}

La Educación Física se integra dentro del modelo psicoeducativo acuñado por Devís y Peiró (1992, citado por Pérez, 2005) y responde a aspectos marcados de forma cualitativa y constructivista, dándole mayor importancia al aspecto formativo e integral del individuo.

López Rodríguez (2003) apunta hacia una serie de corrientes y tendencias pedagógicas que fundamentan las bases teóricas y metodológicas que se van a integrar en este modelo de E.F.; entre ellas, podemos citar el método psicocinético de Jean Le Boulch (1972), la Psicomotricidad relacional de Lapierre y Aucouturier (1976) y la Expresión Corporal (citado por Pérez, 2005).

A raíz de estas nuevas estructuras de pensamiento, aparecen dentro de nuestra área de conocimiento una serie de contenidos explícitos en los currículos educativos relacionados con las manifestaciones básicas de la motricidad humana, donde se le concede una mayor relevancia al conocimiento y al control del propio cuerpo, la comunicación y expresión corporal, las actividades en el medio natural, la actividad física orientada a la salud, las actividades lúdico-recreativas y artísticas...En 
este momento, también se producen nuevas propuestas en base a los contenidos dominantes en el enfoque tradicional, empleando los contenidos de condición física, habilidades y actividades deportivas hacia esquemas de acción que pongan al alumnado en situación de resolver diferentes problemas motores a través del empleo de estrategias y operaciones cognitivas.

Los profesionales de la Educación Física que abogan por un paradigma práctico dan un salto de calidad y se preocupan por dirigir el camino de esta área curricular hacia "la racionalidad práctica orientada a la educación y a la participación" (López, 2006, p. 82).

Al enfoque práctico se le otorga un carácter formativo cuando se genera un conocimiento en el proceso de enseñanza y aprendizaje, para tratar de mejorar y adecuarlo a las circunstancias acaecidas, a través de las interacciones y los momentos que se producen en todo dicho proceso.

Por tanto, se concibe una nueva forma de entender la Educación Física, donde la evaluación no sea confundida con la calificación y se dé luz verde a los procesos de enseñanza, aprendizaje y enseñanza-aprendizaje.

\section{- Fundamentación pedagógica}

Díaz (2005) y López (2006) están de acuerdo en que las claves de los procesos de evaluación se encuentran en los planteamientos de uso y práctica evaluativa; para poder descifrar dichas claves deberemos responder al ¿para qué evaluar? ¿por qué evaluar? y ¿para quién se evalúa?.

¿Para qué evaluar? Este primer interrogante tiene su respuesta en las funciones que ofrecen las prácticas de evaluación en Educación Física en todo el proceso de enseñanza y aprendizaje.

López (1999) establece las funciones de la evaluación desde una racionalidad práctica; así la evaluación huye de la perpetua medición y consecución de resultados, y se interesa por analizar y comprender los pormenores que se producen en el proceso educativo; así, se puede hablar de las siguientes funciones (citado en Díaz, 2005):

- La evaluación como reflexión crítica

- La evaluación como debate crítico y medio de comunicación y diálogo entre alumnos y profesor

- La evaluación como análisis y mejora

- La evaluación como comprensión y mejora

- La evaluación como motor de cambio, perfeccionamiento y mejora profesional

Las funciones se orientan hacia el desarrollo y consecución de una serie de finalidades; así, Sanmartí (2007) considera que las finalidades pedagógicas o reguladoras son propias del paradigma práctico, ya que están relacionadas con identificar los cambios que hay que introducir en el proceso para conseguir mejoras en los procesos de aprendizaje (citado por López, 2009).

Chaparro y Pérez (2010), distinguen tres tipos de evaluación en función de las finalidades y funciones de la evaluación: 
- Evaluación diagnóstica: con la finalidad de observar el nivel de partida inicial no sólo del individuo, sino también de los programas y proyectos que forman parte de los centros y del sistema educativo.

- Evaluación formativa: se orienta al desarrollo del proceso de enseñanza y aprendizaje con intención educativa de mejora.

- Evaluación sumativa: se valoran si se han conseguido los objetivos perseguidos

Esta configuración que nos proponen estas autoras, deben relacionar cada uno de los tres tipos de evaluación, sustentándose la aplicación de una en el desarrollo de la/s anteriores. Por tanto, no se pueden emplear por separado sin tener en cuenta las informaciones y observaciones anteriores.

Desde los planteamientos vertidos surgen una serie de necesidades que responden al ¿Por qué evaluar? donde fundamentalmente se requiere que el proceso de evaluación esté estrechamente vinculado a la participación del alumnado en el mismo.

Para ello es necesario que el sistema y las etapas educativas que comprende, desarrollen una evaluación acorde a un sistema democrático, donde las competencias, los hábitos y las responsabilidades sociales y personales sean fuente de progreso e innovación.

En base a esta idea, López (2009) expone el concepto de evaluación democrática donde los fundamentos de la evaluación se encuentran ligados a unas fuertes implicaciones ético-morales.

La comunidad educativa en general y el personal docente, en particular, deberíamos hacer uso de la evaluación como un instrumento de investigación y formación; de esta manera, la fuente de conocimiento se ampliaría y se enriquecería tanto para el ser humano que aprende y que enseña, con una intención claramente formativa, educativa y democrática.

López (2006) nos alienta hacia la realización de un esfuerzo personal y una responsabilidad profesional en el uso de una práctica de evaluación basada en la formación continua y la indagación coherente y sistematizada; además nos alerta sobre el caso contrario, donde aparecería una "falsa idea de comodidad, falta de interés en innovar y carencia de compromiso personal ante la educación." (p. 63). Desde mi punto de vista, la idea que nos brinda este autor es fundamental para que nuestros esfuerzos lleguen a buen puerto y sean fruto de trabajo individual y colectivo coordinado con los demás agentes educativos (equipo directivo, claustro, familia, equipo de orientación psicopedagógica, inspección educativa,...) e instituciones educativas (centros de formación del profesorado, universidad, seminarios, asociaciones y colegio de Educación Física, publicaciones,...).

En este caso, podemos contestar claramente a la pregunta ¿para quién se evalúa?, ya que el beneficio es global y está interrelacionado. El sistema educativo gozará de plena salud en lo que respecta a la evaluación, gracias a la intención educativa y formativa que se desprende de todo el proceso, donde la participación de los agentes personales y socioeducativos es fundamental. Por tanto, se evalúa para el alumnado, para el profesorado, para la familia, para el equipo directivo, para la inspección educativa,... 
Al igual, que en el paradigma técnico, tenemos que abordar los diversos interrogantes en el modelo alternativo, encontrándonos, como posteriormente veremos, una serie de respuestas que distan mucho de los planteamientos más conservadores.

\section{- Fundamentación técnica}

Respecto al ¿qué evaluar?, dentro de una perspectiva de proceso y proyecto, deberemos evaluar tanto el proceso de enseñanza como el proceso de aprendizaje.

En relación al proceso de enseñanza, aludiremos a tres frases claramente diferenciadas por Hernández y López (2004): "fase pre-activa, fase interactiva y fase post-activa" (p. 58). En primer lugar, el profesorado deberá atender a todas aquellas circunstancias que rodean a la interacción con el alumnado, por lo que deberá tener muy en cuenta los aspectos relacionados con la programación y los niveles de concreción curricular.

Una vez se produzca interacción, se desencadenaran los procesos de enseñanza-aprendizaje donde se produce un triángulo de conocimiento formado por el profesorado, el alumnado y el contexto en el que se desenvuelven. Tras la interacción, es la hora de reflexionar sobre la práctica y el desarrollo de las fases anteriores, para mejorar la puesta en marcha del próximo proceso.

Acerca del proceso de aprendizaje, la acción se encuentra dirigida a la participación del alumnado en la evaluación y en su desarrollo personal y social. Además, al margen de los ámbitos de la personalidad, debemos evaluar el cumplimiento de los objetivos, competencias básicas y contenidos del currículo oficial, utilizando como indicadores los criterios de evaluación.

Nos encontramos, dentro de un planteamiento alternativo donde sus intenciones educativas respecto al objeto de evaluación se encuentran enmarcadas dentro de un contexto determinado y está dinamizado por diferentes agentes educativos que interactúan en las prácticas evaluadoras.

Esta propuesta de evaluación se enmarca dentro de un proyecto educativo, que precisa de diferentes elementos que se tienen que relacionar entre sí. Ello ofrecerá un marco sólido, donde la coherencia y la orientación educativa, son el soporte básico de este modelo de evaluación. Bajo estos menesteres se podrá atender a una serie de condiciones que conseguirán promover y desarrollar una evaluación que sea útil, viable, precisa, individualizada, ética y adecuada a las necesidades, intereses, posibilidades y características del alumnado (Díaz, 2005).

En esta línea cabe preguntarse ¿cómo evaluar?, si realmente queremos estimular el proceso de aprendizaje. Para ello deberemos diseñar en un primer momento una serie de técnicas y después aplicar los instrumentos que orienten la evaluación educativa hacia la consolidación de un proceso basado en el pensamiento crítico, la creatividad, el aprendizaje significativo, la capacidad de análisis y síntesis, la resolución de problemas y la capacidad de esgrimir argumentos razonables y personalizados (Álvarez, 2003).

Para que el registro de la información sea claro, correcto y conciso, Díaz (2005) propone que la "observación debe ser planificada, sistemática, lo más completa posible y registrable" (p. 147). 
Estas intenciones deben plasmarse en una serie de instrumentos, que se ajustarán a un contexto determinado y a unas características individuales del alumnado, que darán lugar a una realidad concreta dentro de la clase de Educación Física. Para reflejar dicha realidad desde un paradigma práctico, podemos diseñar instrumentos tanto para evaluar la enseñanza como el aprendizaje.

Entre la literatura consultada (Díaz, 2005; López, 2006, 2009) encontramos los siguientes instrumentos y técnicas pertenecientes a un discurso práctico entre los profesionales de la Educación Física; en relación a la evaluación del proceso de aprendizaje podemos destacar: el ludograma, el cuaderno del alumno, fichas, trabajos, recensiones, asambleas, puestas en común, la entrevista personal profesor-alumno, la aplicación de las Tecnologías de la Información y de la Comunicación (TIC) en la evaluación de la Educación Física (Web Quests, los deberes Web, Actividades de colaboración en red, la caza del tesoro, los Wiki, los Plan Lesson, los weblog y las actividades colaborativas en red)

Estos procedimientos son mecanismos muy interesantes para desencadenar procesos cognitivos en el alumnado y de observación de aspectos socio afectivos que van a incidir de manera decisiva en la salud psicosocial del alumnado. También implican activamente al alumnado en su participación y le otorga la posibilidad de ser crítico con el trabajo realizado a través de procesos de reflexión, comprensión y mejora. En ocasiones, por el contrario, estas dinámicas restan tiempo al componente motor.

Tras los instrumentos de evaluación del aprendizaje, vamos a desarrollar los instrumentos y técnicas que podemos utilizar en la evaluación del proceso de enseñanza; así podemos destacar: las Escalas de autoevaluación, cuestionarios de evaluación por parte del alumnado,...también la utilización de las TIC pueden ser utilizados por el docente. Por ello, es normal el uso de hojas de cálculo, procesadores de texto y otros programas diseñados expresamente como apoyo al docente de EF; así, autores como Posada (2000) han aportado programas de software educativo como el Efos, el Ludos, y el Cronos.

Es imprescindible que el docente reflexione y recapacite sobre su propia práctica y proporcione herramientas al alumnado para valorar su programa y su actuación docente. De esta manera, la innovación y la evaluación de las propias práctica evaluadoras tienen su concepto en la metaevaluación, donde la contribución de este tipo de evaluación regeneradora producirá una mejora en la calidad de la enseñanza y de los sistemas educativos; en consecuencia, se adquirirán mayores niveles de progreso, bienestar, igualdad y justicia social (Hernández y Velázquez, 2004).

Si nos preguntáramos ¿cuándo evaluar?, la contestación se dirigiría hacia el plano del proceso y el progreso del alumnado a lo largo de un determinado período de tiempo. Este espacio temporal va a gozar de una serie de momentos que van a interrelacionarse en el tiempo con el objetivo de darle coherencia y rigor al proceso de aprendizaje.

Así, podemos hablar de tres momentos en la evaluación: uno inicial, otro continuo y otro final.

Por un lado, la evaluación inicial nos permite apreciar los conocimientos previos del alumnado y realizar las adaptaciones oportunas. 
Por otro lado, la evaluación continua, por su parte, facilita la valoración del desarrollo de los aprendizajes del alumnado mediante la continua recogida de datos e informaciones de cualquier índole. Desde esta vertiente alternativa, se intentan observar y registrar los hándicaps acontecidos, para poder mejorar el proceso.

Y por último, la evaluación final se llevaría a cabo al término de una unidad didáctica, trimestre, curso, ciclo o etapa, donde se comprobará el grado de consecución de los resultados, teniendo en cuenta los otros dos momentos anteriores. Se dará por tanto, desde este enfoque alternativo, una coherencia y una sistematicidad al proceso de aprendizaje a través de su regulación y su capacidad de retroalimentarse.

Según Chaparro y Pérez (2010), esta ordenación de los momentos que acabamos de elaborar, han producido un desconcierto terminológico a la hora de asociar la evaluación inicial a la evaluación diagnóstica, la evaluación procesual a la evaluación formativa, y la evaluación final a la evaluación sumativa; es decir, existe una confusión entre los momentos de la evaluación con sus finalidades en el modelo alternativo.

Una vez respondidas y analizadas las cuestiones al ¿qué evaluar? ¿cómo evaluar? y ¿cuándo evaluar? dentro del modelo alternativo, podemos destacar la confluencia de estas preguntas a través del ¿a quién evaluar? y ¿quién evaluar?

Para justificar dicha convergencia, destacaremos que la participación de los agentes evaluadores en el proceso educativo, que se deben ajustar a una serie de técnicas e instrumentos y en diferentes momento de todo el desarrollo procesual.

En esta ocasión, en el ¿a quién evaluar? la evaluación no sólo se encamina hacia el alumnado como ocurría desde la visión técnica, sino que se centra en el profesorado, el alumnado y los procesos de enseñanza y aprendizaje (López, 2006).

Respecto al ¿quién evaluar? no se produce una unidireccionalidad hacia el proceso de aprendizaje; Chaparro y Pérez (2010) señalan que en función de la persona que evalúa podemos diferenciar entre: heteroevaluación, coevaluación, autoevaluación y evaluación compartida.

Por un lado, la heteroevaluación tiene más cobertura y el alumnado también puede evaluar al profesorado; por otro lado, la coevaluación puede ser entre alumnos, o también entre profesor y alumno, adoptando cada uno diferentes roles y de forma activa en el proceso.

Por su parte, la autoevaluación puede realizarse por el alumnado o por el profesorado, con el fin de provocar un aprendizaje reflexivo para la comprensión de los contenidos llevados a cabo. Fernández (2005) considera la autoevaluación como un pilar fundamental de una educación democrático-humanista basada en la dignidad humana, la cual debe fomentar y defender los derechos y deberes de las personas (citado por Sicilia y Fernández, 2005).

Por último, la evaluación compartida emerge como un proceso dialógico y comunicativo entre el profesorado y el alumnado sobre la evaluación de los aprendizajes y los procesos de enseñanza-aprendizaje.

\section{BIBLIOGRAFÍA}


Álvarez, J.M. (2001). Evaluar para conocer, examinar para excluir. Madrid: Morata.

Álvarez, J.M. (2003). La evaluación a examen ensayos críticos. Madrid: Miño y Dávila.

Blázquez, D. (2003). Evaluar en Educación Física. Barcelona: Inde.

Blázquez, D. y Sebastiani, E. M. (1998). Innovaciones y desafíos en la evaluación de la Educación Física. En Ruiz, F. y Rodríguez, P.L. (Coordinadores). Educación Física, Deporte y Salud (251-282). Universidad de Murcia: Facultad de Educación.

Bores, N. J. y Escudero, E. (1997). La Educación Física del presente a la luz de una evaluación políticamente correcta. Tabanque, 12 (153-170)

Chadwick, C. y Rivera, N. (1991). Evaluación formativa para el docente. Barcelona: Paidós.

Chaparro, F. y Pérez, A. (2010). La evaluación en Educación Física: enfoques tradicionales versus enfoques alternativos. Revista digital Efdeportes, 140. Descargado el 26-12-11 de, http://www.efdeportes.com/efd140/la-evaluacion-eneducacion-fisica-enfoques-alternativos.htm

Contreras, O. R. (2009). Didáctica de la Educación Física (3ae ed.). Un enfoque constructivista. Barcelona: Inde.

Devís, J. y Pérez-Samaniego, V. (2009). La ética profesional en la formación del profesorado de Educación Física. En Ainstein, A.; Martínez, L. y Gómez, R. La educación física y el deporte en la edad escolar: El giro reflexivo en la enseñanza. (pp. 105-128). Buenos Aires: Miño y Dávila.

Díaz, J. (2005). La evaluación formativa como instrumento de aprendizaje en Educación Física. Barcelona: Inde.

Fernández-Balboa, J. M. (2003). Postmodernidad e investigación en la Educación Física. Revista Ágora para la Educación Física y el Deporte, 2-3 (pp. 5-22).

Fernández-Balboa, J.M. (2005). La auto-evaluación como práctica promotora de la democracia y la dignidad. En A. Sicilia y J.M. Fernández Balboa (Coordinadores). La otra cara de la enseñanza: La educación física desde una perspectiva crítica (127-158). Barcelona: Inde.

Gimeno, J. y Pérez, A. I. (1985). La enseñanza: su teoría y su práctica. (2ªed). Madrid: Morata.

Gimeno, J. y Pérez, A. I. (2008). Comprender y transformar la enseñanza. $12^{\underline{a}}$ ed. Madrid: Morata.

Gómez, A.; Díez, L.J.; Fernández, J.M.; Gorrín, A.; Pacheco, J.J. y Sosa, G. (2008). Nueva propuesta curricular para el área de Educación Física en la Educación primaria. Revista Internacional de Medicina y Ciencias de la Actividad Física y el Deporte, 29 (pp. 93-108) http://cdeporte.rediris.es/revista/revista29/artprimaria74.htm

González, M. A. (1995). Manual para la evaluación en Educación Física: Primaria y Secundaria. Madrid: Editorial Escuela Española.

Grundy, S. (1991). Producto o praxis del curriculum. Madrid: Morata.

Hernández, J. L. y López, A. (2004). Evaluación de la enseñanza: análisis y propuestas. En Hernández, J. L. y Velázquez, R. (coords). La evaluación en Educación Física: investigación y práctica en el ámbito escolar. Barcelona: Grao.

Hernández, J.L y Velázquez, R. (2004). La evaluación en educación física. Investigación y práctica en el ámbito escolar. Barcelona: Grao.

Imbernón, F. (1992). Reflexiones sobre la Educación Física y la Reforma educativa. Apunts, 30 (69-70).

Kirk, D. (1990). Educación Física y Currículum. Valencia: Universidad de Valencia.

López, V. M. (2000). Buscando una evaluación formativa en educación física: Análisis crítico de la realidad existente, presentación de una propuesta y análisis general de su puesta en práctica. Revista Apunts: Educación Física y Deportes, 62 (p. 16-26), http://articulos-apunts.edittec.com/62/es/062 016 026 ES.pdf 
López, V. M. (2006). La evaluación en educación física. Revisión de los modelos tradicionales y planteamiento de una alternativa: la evaluación formativa $y$ compartida. Madrid: Miño y Dávila.

López, V. M. (2007). La evaluación en educación física y su relación con la atención a la diversidad del alumnado. Aportaciones, ventajas y posibilidades desde la evaluación formativa y compartida. Revista Kronos, 11, 59-71. Descargado el 27-12-11, http://www.revistakronos.com/docs/File/kronos/11/kronos_11_7.pdf

López, V. M. (2009). Evaluación formativa y compartida en Educación Superior. Madrid: Narcea.

López, V.M., Barba, J.J., Monjas, R., Manrique, J.C.' Heras, C., González, M. y Gómez, J.M. (2007). Trece años de evaluación compartida en Educación Física. Revista Internacional de Medicina y Ciencias de la Actividad Física y el Deporte, 7, 69-86. Descargado el 5 de octubre de 2011, http://cdeporte.rediris.es/revista/revista26/artautoeval48.htm

López, V.M.; Monjas, R. y Pérez, D. (2003). Buscando alternativas a la forma de entender y practicar la Educación Física escolar. Barcelona: Inde.

López, P.A. y Fontana, P. (1998). La utilización de pruebas física como medio calificador: "una herencia patológica". En Actas al II Congreso Internacional sobre la enseñanza de la Educación Física y el deporte escolar. Almería, España.

Ministerio de la Presidencia. Boletín Oficial del Estado núm. 238 de 4/10/1990, p. 28936.http://www.boe.es/aeboe/consultas/bases datos/doc.php?id=BOE-A-199024172 12/10/2011

Ministerio de la Presidencia. Boletín Oficial del Estado núm. 106 de 4/5/2006, pp. 17169-17183.http://www.boe.es/aeboe/consultas/bases datos/doc.php?id=BOE-A2006-7899 12/10/2011

Minkévich, O. (2009). El enfoque cualitativo y la evaluación en Educación Física. En Ainstein, A.; Martínez, L. y Gómez, R. La educación física y el deporte en la edad escolar: El giro reflexivo en la enseñanza. (pp. 285-305). Buenos Aires: Miño y Dávila.

Morales, J. J. (2001). La evaluación en el área de Educación visual y plástica en la Educación Secundaria Obligatoria. Tesis doctoral. Bellaterra: Barcelona. http://www.tesisenred.net/bitstream/handle/10803/5036/jjma01de16.pdf.pdf?sequence= $\underline{1}$

Ordoñez, J. J. (1990). Objetivos en la enseñanza. Causas de un rechazo. Revista Aula abierta, 55 (pp. 89-115).

Pérez, A. (2005). Estudio del planteamiento actitudinal del área de Educación Física de la Educación Secundaria Obligatoria en la LOGSE (una propuesta didáctica centrada en una metodología basada en actitudes). León: Universidad de León.

Posada, F. (2000). Ideas prácticas para la enseñanza de la Educación Física. Lérida: Agonós.

Real Academia Española (2011-2012). Diccionario de la Lengua española: vigésimo segunda edición. Descargado el 7 de octubre de 2011, http://www.rae.es/rae.html

Rosales, C. (1984). Criterios para una evaluación formativa. (2 ${ }^{\underline{a}}$ ed.) Madrid: Narcea.

Sanmartí, N. (2007). (10 ideas clave) Evaluar para aprender. Barcelona: Graó.

Soler, S. y Vilanova, A. (2010). La investigación en Educación Física. En González, C. (coord.) Educación física Investigación, innovación y buenas prácticas. Cap. 2 (pp. 25-42). Barcelona: Grao.

Stenhouse, L. (1984). Investigación y desarrollo del curriculum. Madrid: Morata.

Velázquez, R. (2000). ¿Existe el deporte educativo? Un ensayo en torno a la supuesta naturaleza educativa del deporte. En la formación inicial y permanente del profesor de Educación Física (vol 1). Actas del XVIII Congreso Nacional de Educación Física, 481- 492. Ciudad Real, España. 
Vizuete, M. (2002). La Didáctica de la Educación Física y el área de conocimiento de expresión corporal. Profesores y currículum. Revista de Educación, 328 (pp. 137-154). 